\title{
Enhanced Primary Care improves GP service regularity in elderly patients without impacting on service frequency
}

\section{Abstract}

Objective: Assess impact of Enhanced Primary Care service utilisation on subsequent general practitioner service regularity and frequency.

Methods: A retrospective population-based longitudinal cohort using linked administrative health records of hospital and primary care services for people over the age of 65 years. Multinomial logistic regression modelling was used to evaluate changes in the relative likelihood of increased primary care service regularity and frequency in exposed and unexposed individuals adjusting for age, gender and recent chronic disease hospitalisation history.

Results: Enhanced Primary Care services significantly and substantially increased relative likelihood of increased regularity with no corresponding higher likelihood of increased frequency of general practitioner contact. Increased regularity was more likely with increasing age except for the oldest age group (90+ years). Some chronic disease histories (e.g. diabetes) showed a higher likelihood of improved regularity while others were less likely to produce an increased regularity (e.g. hypertension).

Conclusions: The study suggests a capacity for modification of physician and patient behaviour using incentivised services within the current fee-for-service system in Australia. 


\section{Introduction}

Many developed nations are struggling with the rising cost of avoidable hospitalisations, in Australia two thirds of these admissions are due to conditions considered ambulatory care sensitive chronic diseases (ACSCD) (Parchman and Culler, 1994, Bindman et al., 1995, Basu et al., 2002, Page A et al., 2007). Early disease management and preventative care in a primary care setting are considered effective measures in preventing hospitalisations due to these conditions (Page A et al., 2007). Australia's complex health system involves policy and funding duties spread over federal and state boundaries with service provision split between public and private providers. General practice is largely funded by the federal government using the Medicare Benefits Schedule (MBS) to provide a minimum level of access for all Australians. The federal government is responsible for funding general practice while the federal and state governments both fund the tertiary health system except state governments are responsible for delivery of these services. In November 1999, the Australian federal government implemented the Enhanced Primary Care (EPC) program with the aim of reducing the burden of avoidable hospitalisations from chronic diseases (O'Halloran et al., 2006). This was to be achieved via an increased financial incentive for general practitioners/physicians (GPs) over and above the preexisting fee-for-service payment structure of the MBS which specifies a fee for each service (Department of Health and Ageing, 2009, O’Halloran et al., 2006).

The EPC program comprised a set of service 'items' on the MBS (O'Halloran et al., 2006) designed to improve the regularity and quality of health care provided by GPs to older Australians and those with chronic diseases (O'Halloran et al., 2006, Sims et al., 2000). These items comprised annual health assessments, multidisciplinary care plans and case conferencing reimbursement (Department of Health and Aged Care, 2000-2005). The annual health assessments are limited to patients aged 75 years and over to assess whether preventive or educational services should be offered to the patient (Department of Health and Aged Care). The health assessment was valued at $\$ 149.90$ in the 2001 MBS while a general consult (which would include taking a medical history, physical examination and management plan) was valued by the MBS at 54.60 for the same year (Department of Health and Aged Care, 2000-2005). The multidisciplinary care plans (\$192.75 for 2001 MBS) and case conferences ( $\$ 75.00$ for 15 to 30 minute conference in $2001 \mathrm{MBS}$ ) are for patients of any age with chronic or terminal conditions (Commonwealth Department of 
Health and Aged Care, 1999). The item prices quoted are only used as representative for a range of items with variations of value due to setting and duration of consultation, each year the federal government revises the value of Medicare items to account for changes and incremental inflation.

In November 2004, the EPC program was redesigned and modified after GP feedback, specifically pertaining to the degree of paperwork required to claim the items from the government. The new Chronic Disease Management (CDM) items were less focused on multidisciplinary demands and allowed GPs to produce independent care plans (without consultation or correspondence with other health care practitioners), with a subsequent reduction in value for CDM care plan items compared earlier EPC values (Department of Health and Ageing, 2009, Department of Health and Aged Care, 2000-2005).

EPC items were promoted to Australian GPs to improve chronic disease outcomes (Medicare Australia). In 2007-08, 2.064 million EPC/CDM items were billed nationally at a cost of AUD\$203.8 million (Department of Health and Ageing, 2009). However, evaluation of EPC/CDM services to date has been limited to assessment of awareness and acceptance in GPs (O'Halloran et al., 2006, Blakeman et al., 2001b, Wilkinson et al., 2002a, Cheffins et al., 2010), patient uptake across population subgroups (Wilkinson et al., 2002b, Grimmer-Somers et al., 2010) and program fidelity (Blakeman et al., 2001a, Haines et al., 2010). Evaluation of the programs' impact on regularity and frequency of GP contact and the effects on disease progression and hospitalisations in patients with chronic diseases has thus far not occurred (Department of Health and Ageing, 2009).

The purpose of this study was to assess the influence of utilisation of the EPC/CDM program on GP service regularity and frequency within the same calendar year and to assess the independent effects of demographic factors and chronic disease history.

\section{Methods}

This was a retrospective population-based longitudinal cohort study linking routinely collected administrative health records from the Western Australian data linkage system with Medicare Benefit Schedule records collected and maintained by the Commonwealth Department of Health and Ageing. 


\section{Data sources and study population}

The study population was limited to individuals aged 65+ years who had been registered by Medicare Australia continuously in Western Australia (WA) from 1984 until 2006 (end of the study), death or out-of-state migration (identified using WA Electoral Roll records) (Deeble, 1989). De-identified records for the study population were extracted and linked at the individual level for MBS records originating in WA from 2001-2006, WA hospital morbidity data system (HMDS) records from 1999-2006 and WA mortality records from 2001-2006.

\section{Record selection}

EPC or other GP claim MBS records were identified using item numbers from published Medicare schedules for each year of study. EPC items were defined as any service listed under Groups A14 and A15 of the relevant MBS schedule (Department of Health and Aged Care, 2000-2005). GP claims were defined as any item listed in the explanatory notes of the relevant MBS as 'Attendances by General Practitioners' (Department of Health and Aged Care, 2000-2005). Records not relating to EPC or other GP claims were removed from the MBS dataset (such as allied health professional attendances and diagnostic test billings).

\section{Classification of EPC exposure status}

Individuals were classified as exposed or unexposed to the EPC program each year based on the occurrence of an EPC claim item within each calendar year. Exposure time was determined by the first EPC item claim identified from the MBS data until the end of the calendar year. Individuals were classified as unexposed prior to the first EPC item claim and reverted to unexposed at the start of each subsequent year of the study.

\section{Classification of chronic disease history}

Hospitalisation within two prior years of a first EPC claim (exposed group) or the start of each calendar year (unexposed group) was identified using International Statistical Classification of Diseases and Health Related Problems (ICD) codes for seven ACSCD categories (Caminal et al., 2004) based on the primary and secondary diagnosis fields of HMDS records for each individual. The codes were approved and independently validated 
by a clinical consensus panel comprising nine GPs, two medical specialists and two clinical pharmacists. For each year of the study, an individual was categorised to any of the following eight ACSCD groups: Diabetes, Asthma and Chronic Obstructive Pulmonary Disease (COPD), Ischaemic Heart Disease (IHD), Seizures, Dyspepsia, Hypertension, and Heart Failure or were classified as having no history of chronic disease.

\section{Classification of age}

For each year of the study individuals were classified into one of six age groups ascertained on the date of the first EPC claim (exposed group) or at the start of the calendar year (unexposed group).

\section{Determining the outcome}

1. Regularity of primary care

For each individual, each year was partitioned into time in either the exposed and unexposed state. The MBS data were used to determine the number of days between: (i) the beginning of the exposure status (start of the year for the unexposed state or date of first EPC claim for the exposed state) and the date of the next primary care visit; (ii) number of days between each subsequent primary care visit occurring throughout the calendar year; and (iii) the number of days from the last primary care visit and the end of the calendar year, death or out-of-state migration (whichever occurred first). This provided a distribution of days between visits for each patient in each year. The variance of the distribution was calculated for each individual in each year according to exposure state and the following equation was used to generate a measure of annual primary care service regularity:

$$
R=\frac{1}{(1+\operatorname{Var}(\Phi))}
$$

where R was the regularity and $\operatorname{Var}(\Phi)$ was the variance of the days between visits.

By calculating regularity as above, a score from 0 to 1 is possible, with a score of 1 indicating perfect regularity of services. This method of calculating service regularity has been reported previously (Gill and Mainous, 1998, Gill et al., 2000). The measure differs from a simple frequency count of services and requires at least two services in a single 
calendar year to provide a score, thus individuals with less than two service records in any calendar year were removed from the analysis.

\section{Frequency of primary care service}

For each individual, each year was partitioned into time in both the exposed and unexposed state, and the total number of MBS primary care visits (GP or EPC claims) for each individual for each year was enumerated. Frequency scores were reported as an integer having a range of 0 to 365 .

3. Conversion of score into quintiles of regularity and frequency

To provide a relative comparison of regularity and frequency, scores were converted to quintiles (lowest, low, medium, high and highest) based on the range of scores observed in the entire study cohort. Since the distribution of scores in both regularity and frequency were highly skewed, quintiles of regularity and frequency were generated using the log median and two standard deviations of the values in each respective score.

Modelling the likelihood of increased GP service regularity and frequency according to exposure to the EPC program

Multinomial logistic regression modelling was used to evaluate changes in the likelihood of increased (i) GP service regularity and (ii) frequency of GP service within the remainder of a single calendar year in individuals exposed to the EPC program compared with the unexposed individuals adjusting for age, gender and recent chronic disease history. The correlated data structure (repeated observations on each individual) required use of a random effects model so the relative rate ratio of regularity or frequency increase could be modelled explicitly for each individual in a particular calendar year taking into account their exposure status in other calendar years (Hosmer and Lemeshow, 1989, Carriere and Bouyer, 2004). Similar models were also constructed separately for each year of the study period so changes in likelihood over time could be evaluated. In addition, to evaluate the independent effects of gender, age and chronic disease history on the likelihood of increased GP service regularity and frequency across the study period, multinomial logistic regression modelling was undertaken as described above limited to exposed individuals. 


\section{Results}

As shown in table 1, the largest increase in the number and proportion of individuals exposed to the EPC/CDM program occurred in 2005 when the proportion increased from $9.1 \%$ to $14.1 \%$. While there was little change in the gender of individuals exposed to the EPC/CDM program across the study period, with females consistently accounting for a slightly higher proportion of the study population, changes in the age profile of exposed individuals were noted. Over time, there was a decline in the proportion of individuals aged 65 - 69 years (approximately 29\% from 2001 to 2004 dropping to $25.8 \%$ in 2005 and $20.4 \%$ in 2006) and an increase in individuals aged 70 - 84 years. For both regularity and frequency, all study years showed a slightly higher proportion of individuals in the medium quintile and smaller numbers at the more extreme ranges. However, the lowest frequency quintile averaged a higher proportion of individuals than the lowest regularity quintile, $18.2 \%$ compared to $12.6 \%$ respectively.

Figure 1 shows the result of the models estimating the likelihood of an increase in regularity and the likelihood of an increase in frequency according to exposure to the EPC program adjusted for gender, age, recent chronic disease history and year of exposure. In the figure, each bar represents the risk ratio (likelihood) of an exposed individual having the depicted quintile of regularity or frequency compared with the baseline (lowest quintile) relative to the risk ratio (likelihood) of the same scenario in unexposed individuals. In other words, the bars represent the relative difference in likelihood across the two groups. Thus, a relative risk ratio of 1 indicates no difference in likelihood of having that quintile of regularity or frequency across the exposure groups, less than 1 indicates a reduced likelihood in the exposed group and a value of greater than 1 indicates a higher likelihood in the exposed group.

With respect to regularity, those exposed to the EPC program were consistently more likely to have a higher quintile of regularity compared with those not exposed to the EPC program. In addition, the increase in likelihood grew with each subsequent rise in quintile, indicating the difference between the exposed and unexposed was greatest when comparing highest against lowest regularity. As shown in figure 1, exposed individuals were approximately 14 times more likely to be classified with highest rather than lowest regularity compared with their unexposed counterparts. Conversely, with respect to 
frequency, exposed individuals were consistently marginally less likely to have a higher quintile of frequency compared with their unexposed counterparts, with no significant change in the relative likelihood observed with rise in quintile. These results indicate exposure to the EPC program significantly increased regularity and slightly (but significantly) reduced frequency of GP contact.

Figure 2 shows the result of modelling the likelihood of an increase in regularity according to exposure to the EPC program adjusted for gender, age, recent chronic disease history separately for each year of the study period. Overall, a similar trend was observed as presented in Figure 1, with those exposed to the EPC program consistently having an increasing likelihood of higher regularity compared to their unexposed counterparts. This increase in regularity became larger with each subsequent year until 2006, across all quintiles, with the notable exceptions of the highest quintile where a reduction in the relative increase in likelihood was observed in 2002.

Notably, the increased likelihood observed in the highest regularity quintile for 2005 was of a much greater magnitude than in previous years, with exposed individuals 21.65 (19.80, $23.6895 \%$ C.I.) times more likely to be classified with highest regularity compared with their unexposed counterparts. This phenomenon was also observed to a lesser extent in the high quintile. Across all quintiles, a slight reduction in the relative increase in likelihood of higher regularity was observed for 2006.

Figure 3 shows the independent effect of age on the likelihood of an increase in regularity for those exposed to the EPC program in the age group indicated compared with the likelihood in the youngest age group (65-69 years). The results indicate age is a significant independent factor affecting the degree of increase in regularity afforded by exposure to the EPC program. Compared with the youngest age group, the likelihood of those exposed to the EPC program being classified with low, medium or high regularity increases with age up to age 79 following which it decreases. However, the likelihood of those exposed to the EPC program being classified as highest increases with age up to age 89, following which it declines. 
Figure 4 shows the independent effect of previous hospitalisation for chronic disease on the likelihood of an increase in regularity classification (from lowest) for those exposed to the EPC program compared with the likelihood in those without such a history. Individuals having a previous hospitalisation for diabetes, IHD, angina and dyspepsia all showed an increasing trend to suggest these histories are associated with exposure to the EPC program, affording improved regularity compared with their counterparts who had no chronic disease hospitalisation within the previous two years. In contrast, individuals with

a history of hypertension showed a statistically significant reduced likelihood of being in a regularity quintile above the lowest compared to those with no history, while those with heart failure, and asthma/COPD only appeared to be associated with an increased likelihood of improved regularity for the highest quintile.

\section{Discussion}

A recent report from the Commonwealth Department of Health and Ageing identified a lack of evidence for the effects of the EPC/CDM programs despite their \$203.8 million per year cost (Department of Health and Ageing, 2009). This study has shown exposure to EPC services in WA is strongly associated with an increased relative regularity of GP services during the year of exposure in a consistent manner across quintiles. Further we have shown that this association is not the result of frequency alone, which did not produce any relationship with EPC exposure. This finding suggests EPC items in the Australian Medicare schedule are an effective encouragement for improved regularity of GP services, an important component of how these items were intended to reduce the burden of ACSCDs (O'Halloran et al., 2006). Since evidence indicates that a relative increase in GP service regularity reduces mortality risk (Einarsdóttir et al., 2010) the results of our study suggest that these EPC items may be a cost-effective approach to reducing the burden of chronic disease.

When comparing the likelihood of exposure to the EPC system being associated with higher regularity across time, individuals exposed in 2005 had a much greater likelihood of higher regularity for the remainder of the year than those exposed in any other year. This spike is correlated with the first full calendar year after the changes made to the EPC items and the creation of CDM items (new MBS schedules are implemented in November of each year with items changes and price adjustments). The changes made to the EPC 
items were publicised as measures to reduce the paperwork GPs were required to complete (Department of Health and Ageing, 2009). GP efforts to use the items after the modifications may have increased but gradually washed out in 2006, which raises questions regarding long term utilisation by GPs and capacity for continued use. The extent to which EPC and CDM items have been utilised by Australian GPs has been a concern and was the impetus behind the changes made to the system. In 2006/07, of GPs who claimed 1,000 or more services, $86 \%$ used the care plan items; however, only $34 \%$ of these claimed them more than 50 times year, and the 10\% of GPs who claimed the most care plans accounted for 54\% of all care plans claimed (Department of Health and Ageing, 2009) indicating that utilisation is clustered rather than systematic.

The reason for GP utilisation of these incentives has been shown to be strongly associated with the practice the GP operates from and its capacity to use the EPC/CDM items (Proudfoot et al., 2007). Following this finding the Professional Services Review (PSR) expressed concern that practices with the greatest capacity to use these higher rebated items are at risk of utilising the items on a business basis rather than under clinical motivations (Professional Services Review, 2006). The main concern being that care plans are generated based on practice management driven formulas without considering the patient's needs (Professional Services Review, 2006).

Issues of opportunistic billing could be further exacerbated by the lack of any real outcome measure built into the EPC/CDM items, although such a requirement contradicts the goals of the CDM review in reducing bureaucratic red tape (Department of Health and Ageing, 2009). Given our finding that the 2006 regularity was not statistically significantly different from the years prior to 2005 , the CDM review may not have ultimately improved the program's effect. This finding could justify a reversion to a multidisciplinary focus for the items in order to improve outcomes and avoid exploitative billing practices, which the Department of Health and Ageing recommended in their "Report to Support Australia's First National Primary Health Care Strategy" (Department of Health and Ageing, 2009). However, to date strong direct evidence that these items improve outcomes such as avoidable hospitalisations is lacking, although there is some evidence increased primary care regularity reduces mortality (Einarsdóttir et al., 2010). We intend to make use of these 
data extractions to analyse the impact of EPC and CDM item on the outcomes of the WA population.

When limiting the model to only patients who had used EPC items, we found a variation between age groups. With a reference age group of $65-69$ years, there was a statistically significantly greater likelihood of higher regularity for all but patients in the oldest age group, which were consistently less likely to have a higher regularity than the youngest patients under study. These results suggest that exposure to the EPC system affords improvement of GP service regularity for older patients until the age of 90, at which the effect is lost. This may represent a shift in the health service demands of patients in this age group; however, the literature is unclear about the specific health service utilisation needs of people aged over 90 years in comparison to relatively younger seniors since most studies do not compartmentalise the senior patients beyond a catch-all 65 or 75 years and over. The reduced relative regularity of the patients aged over 90 years exposed to EPC items could also be an artefact of a latently higher regularity for these patients prior to EPC exposure. However, a comparison of the mean regularities of the those over 90 years prior to exposure did not support this hypothesis. Given the evidence regarding the increase in hospital costs in the final years of life, it could be argued that patients aged over 90 years have a greater propensity to require acute care (Moorin and Holman, 2007, Zweifel et al., 1999, Zweifel et al., 2004).

Our study showed that some chronic disease histories responded more favourably and unfavourably for improved regularity in comparison to patients without a recent history of the seven identified ACSCDs. Variations in improvement in regularity, according to chronic disease history is an important policy finding, since it indicates diseases likely to be the most cost-effective responders to exposure to the EPC/CDM programs. The low response of hypertension and heart failure to EPC exposure was unexpected and may be the product of GP preference relating to the management of these conditions. Those with a recent history of Asthma and COPD were also less likely to have an improved regularity. One explanation for this finding in asthmatics is the existence of specific asthma management items in the Medicare schedule and other programs targeted at GPs such the release of an Asthma Management Handbook, an "Asthma 3+ Visit Plan” program, as well as the Asthma Action Plan (Barton et al., 2008, Glasgow, 2008). These programs gained 
prominence following the inclusion of Asthma in the National Health Priority Areas in 1999 (Australian Institute of Health and Welfare (AIHW), 2008). Since our study evaluated the 'relative' effect of the EPC program on regularity we would not expect a significant increase if regularity was already at optimal levels. Currently, COPD is heavily under-diagnosed and under-treated in Australia and there are no specific COPD items operating in the MBS (Glasgow, 2008, Access Economics, 2008). Thus a similar explanation for the reduced effect of the EPC/CDM system for patients with COPD as that put forward for asthma is lacking. Given each chronic disease and patient is likely to involve an array of differing demands and requirements on the primary care system it is unlikely broad items such as the EPC/CDM will ever remove the need for disease specific items.

\section{Strengths and limitations}

This study is unique in its treatment of regularity and frequency separately to determine which is achieved by the program under study. This distinction is crucial in determining if regularity can serve as an interim outcome in assessing the success of primary care programs for chronic disease management. The nature of the regularity formula allows for distinction between individuals with similar frequencies of service utilisation but with different distributions (e.g. a cluster of five visits over a month after a serious event related to the patients' prevalent chronic disease or five evenly spaced structured visits as part of a chronic disease care plan and health assessment routine throughout a 12 month period).

There is a low proportion of missing data values $(<1 \%)$ in data from the WADLS and the specificity and sensitivity of linkage between records is extremely high (>99\%). This is achieved with the combined effect of probabilistic matching, clerical checking and chains of links covering up to 40 years of records for some individuals (Holman, 1999). Except diagnosis fields contained within the HMDS (using ICD coding), administrative data is subject to limited clinical information. Thus, our evaluation of previous history of chronic disease was based entirely on prior hospitalisation for those specific conditions. Consequently, the comparisons of patients with a chronic disease history and patients without is somewhat skewed towards those who are more seriously ill (actually hospitalised). The patients we categorised as lacking a history of chronic disease may have 
been diagnosed with a chronic disease we were examining but did not require hospitalisation in the previous two years. However, we feel this comparison is still valuable in assessing the impact of the EPC/CDM Medicare items because the overall purpose of the items is to prevent avoidable hospitalisations and improve quality of life for these patients.

Socio-economic status and locational accessibility information was present in both the HMDS and mortality records, however the MBS records (which provided information on total GP and EPC claims) did not contain this information. It is possible some of the effects we observed were confounded by socio-economic status, which is a known confounder of health outcomes. Consequently, we cannot comment on the potential impact of restricting or increasing the EPC/CDM items according to socio-economic status. In order to ensure our analysis accounted for socio-economic status and locational accessibility, our study population would need to be restricted to only individuals with a HMDS or mortality record. This restriction would limit the scope of our assessment of a national population-based program to only individuals who had been hospitalised or died during the study period.

The use of the linked MBS, HMDS, Electoral Roll and mortality records, providing comprehensive, longitudinal coverage of health service utilisation and censoring dates for each patient during the study period, was a major strength of the analysis. The administrative data is collected in a routine manner, meaning it is unlikely to be influenced by the process of data collection itself. The population selection, all permanent WA residents born prior to 1940, maximised the external validity of our results. Since the study did not rely on self-reporting, information bias arising from these sources was also minimised.

\section{Conclusion}

This study has demonstrated exposure to EPC/CDM services in Australia increased subsequent regularity for the remainder of the calendar year independent of frequency of service utilisation. This study indicates the potential to influence GP utilisation patterns in a fee-for-service setting with a service provider orientated additional incentive program. Our finding that the effect of exposure varied across both age groups and recent history 
chronic disease hospitalisation suggests that EPC/CDM programs do not hold uniform value. This has implications for cost-effectiveness and suggest that the items should be targeted more strategically. Crucially, this research provides evidence that exposure to EPC/CDM services increases the regularity of GP services, an outcome thought to be associated inversely with chronic disease progression. However, further empirical evidence of this relationship between EPC/CDM items is required in addition to costeffective analyses to inform future policy decisions for the program. While the EPC/CDM programs provide extra incentive for the already present fee-for-service system currently in operation in Australia this type of program could be adopted in other systems under an additional incentive or bonus payment.

\section{Acknowledgements}

This research was funded by a project grant from the National Health and Medical Research Council of Australia. We thank the Commonwealth Department of Health and Ageing and the WA Department of Health for the provision of the linked records.

\section{Conflicts of Interest}

The authors of the paper have no conflicts of interest to declare relating to this study. 
Table 1 Number and percentage of exposed individuals, regularity quintiles, frequency quintiles and patient demographics 2001 to 2006.

\begin{tabular}{|c|c|c|c|c|c|c|c|c|c|c|c|c|c|c|c|}
\hline & & \multicolumn{12}{|c|}{ Year of MBS service } & \multirow{2}{*}{\multicolumn{2}{|c|}{ Total }} \\
\hline & & \multicolumn{2}{|c|}{2001} & \multicolumn{2}{|c|}{2002} & \multicolumn{2}{|c|}{2003} & \multicolumn{2}{|c|}{2004} & \multicolumn{2}{|c|}{2005} & \multicolumn{2}{|c|}{2006} & & \\
\hline & & $\mathrm{N}$ & $\%$ & $\mathrm{n}$ & $\%$ & $\mathrm{n}$ & $\%$ & $\mathrm{n}$ & $\%$ & $\mathrm{n}$ & $\%$ & $\mathrm{n}$ & $\%$ & $\mathrm{n}$ & $\%$ \\
\hline \multirow{3}{*}{$\begin{array}{l}\text { Exposed } \\
\text { Individuals }\end{array}$} & Exposed & 12,678 & 7.3 & 16,232 & 9.0 & 15,615 & 8.5 & 17,409 & 9.1 & 26,235 & 14.1 & 34,907 & 19.6 & 123,076 & 11.3 \\
\hline & Unexposed & 161,024 & 92.7 & 163,169 & 91.0 & 169,132 & 91.5 & 174,148 & 90.9 & 159,810 & 85.9 & 143,563 & 80.4 & 970,846 & 88.7 \\
\hline & Total & 173,702 & 100 & 179,401 & 100 & 184,747 & 100 & 191,557 & 100 & 186,045 & 100 & 178,470 & 100 & $1,093,922$ & 100 \\
\hline \multirow[t]{3}{*}{ Gender } & Male & 73,968 & 42.6 & 77,084 & 43.0 & 80,150 & 43.4 & 84,218 & 44.0 & 81,967 & 44.1 & 78,338 & 43.9 & 475,725 & 43.5 \\
\hline & Female & 99,734 & 57.4 & 102,317 & 57.0 & 104,597 & 56.6 & 107,339 & 56.0 & 104,078 & 55.9 & 100,132 & 56.1 & 618,197 & 56.5 \\
\hline & Total & 173,702 & 100 & 179,401 & 100 & 184,747 & 100 & 191,557 & 100 & 186,045 & 100 & 178,470 & 100 & $1,093,922$ & 100 \\
\hline \multirow{7}{*}{$\begin{array}{l}\text { Age group } \\
\text { (years) }\end{array}$} & $65-69$ & 50,837 & 29.3 & 52,368 & 29.2 & 54,564 & 29.5 & 56,769 & 29.6 & 47,910 & 25.8 & 36,324 & 20.4 & 298,772 & 27.3 \\
\hline & $70-74$ & 47,688 & 27.5 & 48,205 & 26.9 & 48,253 & 26.1 & 48,568 & 25.4 & 48,505 & 26.1 & 49,911 & 28.0 & 291,130 & 26.6 \\
\hline & $75-79$ & 33,954 & 19.5 & 36,019 & 20.1 & 37,715 & 20.4 & 39,604 & 20.7 & 41,203 & 22.2 & 41,740 & 23.4 & 230,235 & 21.1 \\
\hline & $80-84$ & 20,650 & 11.9 & 22,133 & 12.3 & 23,345 & 12.6 & 25,080 & 13.1 & 25,574 & 13.8 & 27,095 & 15.2 & 143,877 & 13.2 \\
\hline & $85-89$ & 13,449 & 7.7 & 13,170 & 7.3 & 12,928 & 7.0 & 12,926 & 6.8 & 13,685 & 7.4 & 14,194 & 8.0 & 80,352 & 7.3 \\
\hline & $90+$ & 7,124 & 4.1 & 7,484 & 4.2 & 7,910 & 4.3 & 8,541 & 4.5 & 8,995 & 4.8 & 9,173 & 5.1 & 49,227 & 4.5 \\
\hline & Total & 173,702 & 100 & 179,379 & 100 & 184,715 & 100 & 191,488 & 100 & 185,872 & 100 & 178,437 & 100 & $1,093,593$ & 100 \\
\hline \multirow{6}{*}{$\begin{array}{l}\text { Regularity } \\
\text { quintile }\end{array}$} & Lowest & 24,060 & 13.9 & 24,244 & 13.5 & 25,010 & 13.5 & 25,205 & 13.2 & 20,140 & 10.8 & 19,011 & 10.7 & 137,670 & 12.6 \\
\hline & Low & 38,180 & 22.0 & 39,164 & 21.8 & 41,270 & 22.3 & 42,295 & 22.1 & 40,980 & 22.0 & 38,438 & 21.5 & 240,327 & 22.0 \\
\hline & Medium & 53,216 & 30.6 & 56,190 & 31.3 & 58,231 & 31.5 & 60,921 & 31.8 & 60,032 & 32.3 & 57,720 & 32.3 & 346,310 & 31.7 \\
\hline & High & 37,793 & 21.8 & 39,499 & 22.0 & 39,848 & 21.6 & 41,864 & 21.9 & 41,633 & 22.4 & 41,121 & 23.0 & 241,758 & 22.1 \\
\hline & Highest & 20,453 & 11.8 & 20,282 & 11.3 & 20,356 & 11.0 & 21,203 & 11.1 & 23,087 & 12.4 & 22,147 & 12.4 & 127,528 & 11.7 \\
\hline & Total & 173,702 & 100 & 179,379 & 100 & 184,715 & 100 & 191,488 & 100 & 185,872 & 100 & 178,437 & 100 & $1,093,593$ & 100 \\
\hline \multirow{6}{*}{$\begin{array}{l}\text { Frequency } \\
\text { Quintile }\end{array}$} & Lowest & 30,227 & 17.4 & 31,748 & 17.7 & 33,255 & 18.0 & 34,229 & 17.9 & 34,350 & 18.5 & 34,982 & 19.6 & 198,791 & 18.2 \\
\hline & Low & 30,363 & 17.5 & 31,814 & 17.7 & 33,301 & 18.0 & 34,936 & 18.2 & 35,041 & 18.9 & 34,177 & 19.2 & 199,632 & 18.3 \\
\hline & Medium & 47,947 & 27.6 & 49,806 & 27.8 & 51,627 & 27.9 & 53,687 & 28.0 & 53,003 & 28.5 & 50,715 & 28.4 & 306,785 & 28.1 \\
\hline & High & 42,202 & 24.3 & 43,253 & 24.1 & 43,914 & 23.8 & 45,349 & 23.7 & 42,809 & 23.0 & 40,092 & 22.5 & 257,619 & 23.6 \\
\hline & Highest & 22,963 & 13.2 & 22,758 & 12.7 & 22,618 & 12.2 & 23,287 & 12.2 & 20,669 & 11.1 & 18,471 & 10.4 & 130,766 & 12.0 \\
\hline & Total & 173,702 & 100 & 179,379 & 100 & 184,715 & 100 & 191,488 & 100 & 185,872 & 100 & 178,437 & 100 & $1,093,593$ & 100 \\
\hline
\end{tabular}




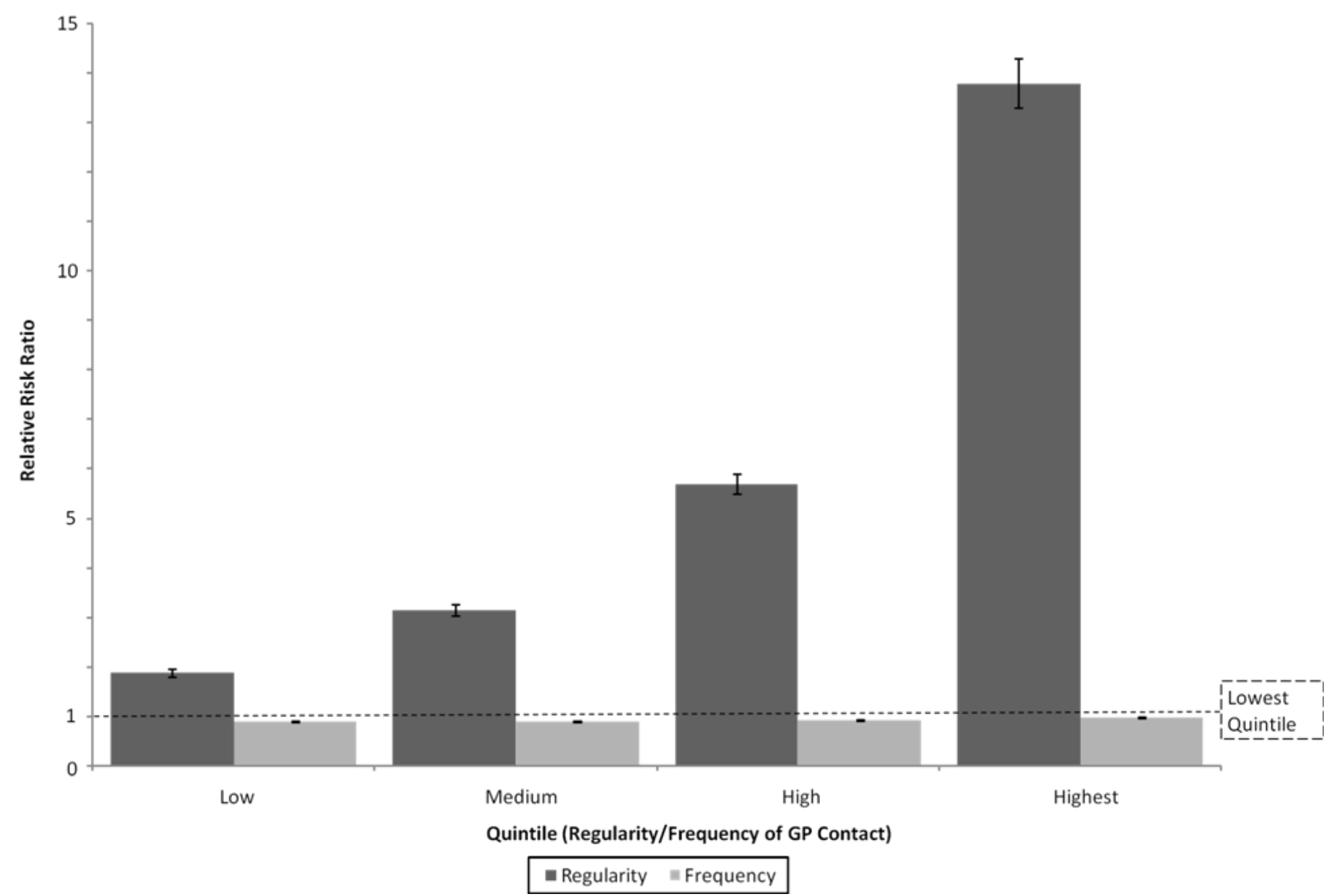

Figure 1 Adjusted* likelihood of increased (i) regularity and (ii) frequency for individuals exposed to the EPC program relative to their non-exposed counterparts.

*Both models are adjusted for age group, gender, year and chronic disease history. 


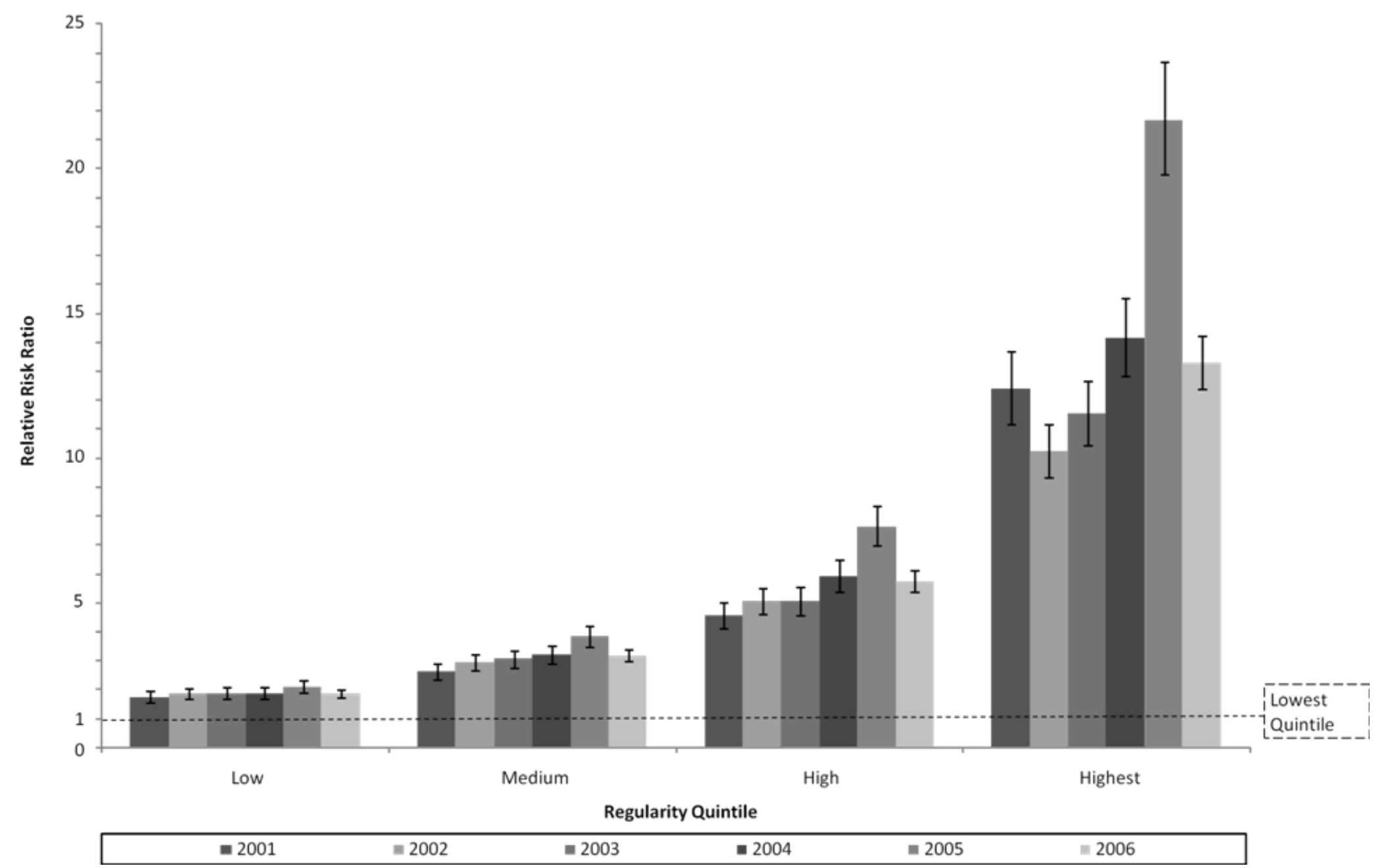

Figure 2 Adjusted* likelihood of increased regularity for individuals exposed to the EPC program relative to their non-exposed counterparts according to year of exposure

*Each year model are adjusted for gender, age group and chronic disease history. 


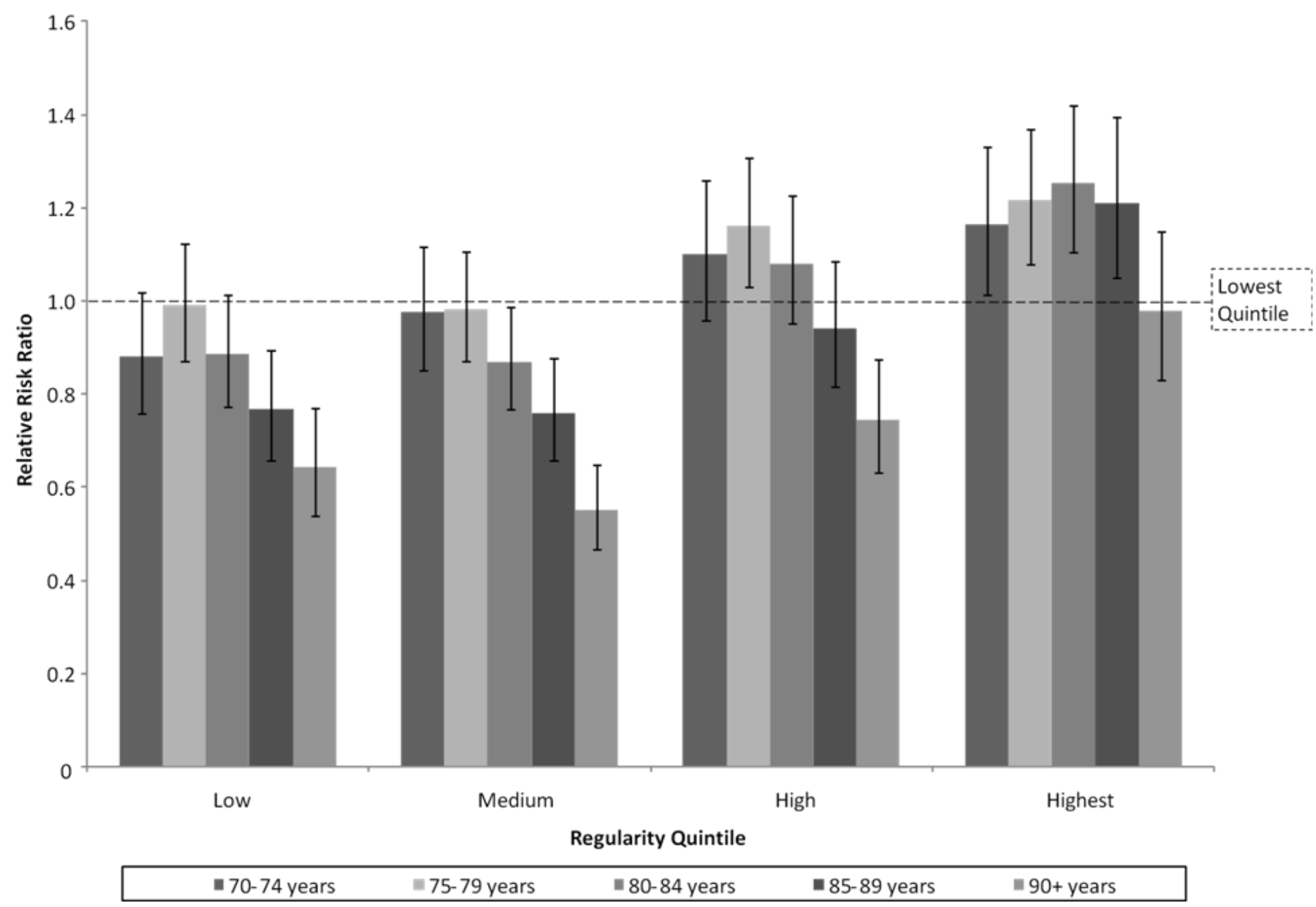

Figure 3 Changes in the adjusted* likelihood of increased regularity for individuals exposed to the EPC program according to age at exposure

*Age group models adjusted for gender, year and chronic disease history. 


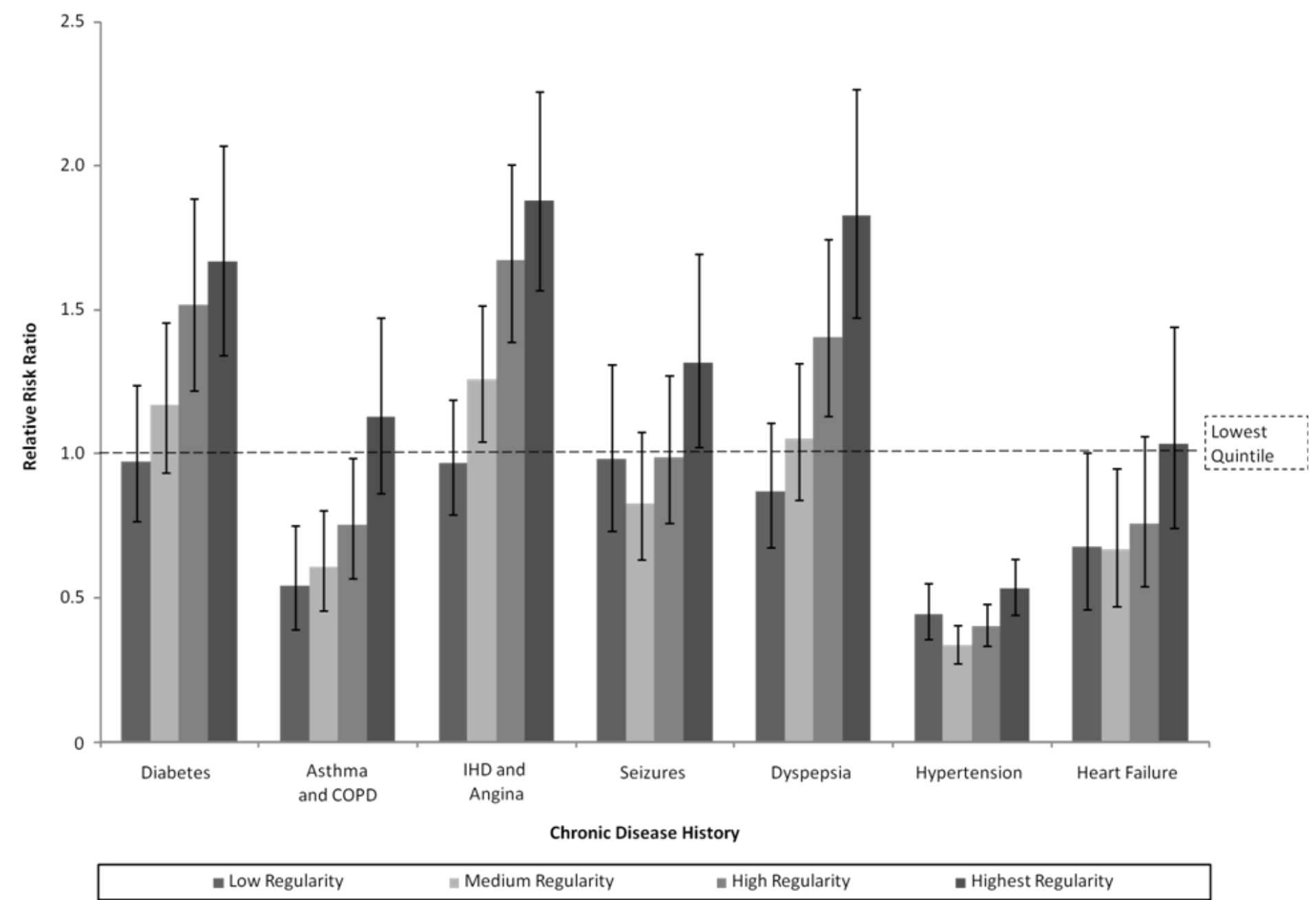

Figure 4 Changes in the adjusted* likelihood of increased regularity for individuals exposed to the EPC program according to previous history of hospitalisation for chronic disease

*Chronic disease history models adjusted for gender, age group and year. 


\section{References}

Access Economics 2008. Economic impact of COPD and cost effective solutions. The Australian Lung Foundation.

Australian Institute of Health and Welfare (Aihw). 2008. Health Priority Areas [Online]. Canberra: AIHW. Available: http://www.aihw.gov.au/nhpa/ [Accessed 25/2/2010].

Barton, C., Proudfoot, J., Amoroso, C., Ramsay, E., Holton, C., Bubner, T., Harris, M. \& Beilby, J. 2008. Management of asthma in Australian general practice: care is still not in line with clinical practice guidelines. Prim Care Resp J, 18, 100-105.

Basu, J., Friedman, B. \& Burstin, H. 2002. Primary care, HMO enrollment, and hospitalization for ambulatory care sensitive conditions - A new approach. Med Care, 40, 1260-1269.

Bindman, A. B., Grumbach, K., Osmond, D., Komaromy, M., Vranizan, K., Lurie, N., Billings, J. \& Stewart, A. 1995. Preventable Hospitalizations and Access to HealthCare. JAMA, 274, 305-311.

Blakeman, T., Harris, M., Comino, E. \& Zwar, N. 2001a. Implementation of the enhanced primary care items requires ongoing education and evaluation. Aust Fam Physician, 30, 75.

Blakeman, T. M., Harris, M. F., Comino, E. J. \& Zwar, N. A. 2001b. Evaluating general practitioners' views about the implementation of the Enhanced Primary Care Medicare items. Med J Aust 175, 95-98.

Caminal, J., Starfield, B., Sanchez, E., Casanova, C. \& Morales, M. 2004. The role of primary care in preventing ambulatory care sensitive conditions. Eur J of Public Health, 14, 246-251.

Carriere, I. \& Bouyer, J. 2004. Choosing Marginal or Random-Effects Models for Longitudinal Binary Responses: Application to Self-Reported Disability among Older Persons [Online]. Available: http://www.biomedcentral.com/14712288/2/15 [Accessed].

Cheffins, T., Spillman, M., Heal, C., Kimber, D., Brittain, M. \& Lees, M. 2010. Evaluating the use of Enhanced Primary Care Health Assessments by general practices in north Queensland. Aust J Prim Health, 16, 221-223.

Commonwealth Department of Health and Aged Care 1999. Medicare Benefits Schedule Book, Canberra, DHAC.

Deeble, J. 1989. Health Care Under Universal Insurance: The First Three Years of Medicare. In: Butler, J. R. G. \& Doessel, D. P. (eds.) Health Economics: Australian Readings. Sydney: Australian Professional Publications.

Department of Health and Aged Care. Older Persons Health Assessment [Online]. DoHA. Available: http://www.health.gov.au/internet/main/publishing.nsf/Content/healthepc-hlthassmnt-factsheet [Accessed 22/04/2009].

Department of Health and Aged Care 2000-2005. Medicare Benefits Schedule, Canberra, DoHA.

Department of Health and Ageing 2009. Primary Health Care Reform in Australia: Report to Support Australia's First National Primary Health Care Strategy. Canberra: DoHA.

Einarsdóttir, K., Preen, D. B., Emery, J. D. \& Holman, C. D. J. 2010. Regular Primary Care Decreases the Likelihood of Mortality in Older People with Epilepsy. Med Care, In Press.

Gill, J. M. \& Mainous, A. G. 1998. The role of provider continuity in preventing hospitalizations. Arch Fam Med 7, 352-357. 
Gill, J. M., Mainous, A. G. \& Nsereko, M. 2000. The effect of continuity of care on emergency department use. Arch Fam Med 9, 333-338.

Glasgow, N. 2008. Systems for the management of respiratory disease in primary care - an international series: Australia. Prim Care Resp J, 17, 19-25.

Grimmer-Somers, K., Dolesj, W. \& Atkinson, J. 2010. Enhanced Primary Care pilot program benefits Type II diabetes patients. Aust Health Rev, 34, 18-24.

Haines, T., Foster, M., Cornwell, P., Fleming, J., Tweedy, S., Hart, A. \& Mitchell, G. 2010. Impact of Enhanced Primary Care on equitable access to and economic efficiency of allied health services: a qualitative investigation. Aust Health Rev, 34, $30-35$.

Holman, C. D. J. 1999. Western Australia:Development of a health services research linked database. Aust N Z J Public Health 23, 453-459.

Hosmer, D. \& Lemeshow, S. 1989. Applied Logistic Regression, New York, John Wiley \& Sons Inc.

Medicare Australia. Medicare Benefits Schedule (MBS) Item Statistics Reports [Online]. Health Insurance Commission. Available:

https://www.medicareaustralia.gov.au/statistics/mbs_item.shtml [Accessed 22/4/2009].

Moorin, R. \& Holman, C. 2007. The cost of in-patient care in Western Australia in the last years of life: A population-based data linkage study. Health Pol, 85, 284 - 295.

O'halloran, J., Ng, A., Britt, H. \& Charles, J. 2006. EPC encounters in Australian general practice. Aust Fam Physician 35, 8-10.

Page A, Ambrose S, Glover J \& D., H. 2007. Atlas of Avoidable Hospitalisations in Australia: ambulatory

care-sensitive conditions [Online]. Adelaide: The University of Adelaide. Available: http://www.publichealth.gov.au/pdf/atlases/avoid_hosp_aust_2007/avoid_hosp_ful 1.pdf [Accessed].

Parchman, M. \& Culler, S. 1994. Primary care physicians and avoidable hospialisations. $J$ Fam Prac, 39, 123-8.

Professional Services Review. 2006. PSR Annual Report 2005-2006 [Online]. Available: http://www.psr.gov.au/docs/publications/html05-06/directors-report.html [Accessed Feb 2010].

Proudfoot, J., Infante, F., Holton, C., Powell-Davies, G., Bubner, T., Beilby, J. \& Harris, M. 2007. Organisational capacity and chronic disease care - An Australian general practice perspective. Aust Fam Physician, Vol 35, 286-88.

Sims, J., Kerse, N. M., Naccarella, L. \& Long, H. 2000. Health promotion and older people: the role of the general practitioner in Australia in promoting healthy ageing. Aust N Z J Public Health 24, 356-359.

Wilkinson, D., Mcelroy, H., Beilby, J., Mott, K., Price, K., Morey, S. \& Best, J. 2002a. Uptake of health assessments, care plans and case conferences by general practitioners through the Enhanced Primary Care program between November 1999 and October 2001. Aust Health Rev, 25, 1.

Wilkinson, D., Mcelroy, H., Beilby, J., Mott, K., Price, K., Morey, S. \& Best, J. 2002b. Variation in levels of uptake of Enhanced Primary Care item numbers between rural and urban settings, November 1999 to October 2001. Aust Health Rev, 25, 123.

Zweifel, P., Felder, S. \& Meiers, M. 1999. Ageing of population and health care expenditure: A red herring? Health Econ, 8, 485-496.

Zweifel, P., Felder, S. \& Werblow, A. 2004. Population ageing and health care expenditure: New evidence on the "red herring". Geneva Pap R I, 29, 652-666. 
